\title{
Collinsella intestinalis
}

National Cancer Institute

\section{Source}

National Cancer Institute. Collinsella intestinalis. NCI Thesaurus. Code C86302.

A species of anaerobic, Gram positive, rod shaped bacterium in the phylum

Actinobacteria. This species is leucine arylamidase positive, lipase and trypsin negative,

nonsporulating, non-motile, produces acid from ribose, glucose, mannose, cellobiose and salicin but not from maltose, lactose, trehalose, esculin or amygdalin. C. intestinalis is part of the intestinal microflora of humans and animals and is not considered to be pathogenic. 